\title{
Cosmological Implications of Neutrinos
}

\author{
A.D. Dolgov \\ INFN, sezzione di Ferrara, via Paradiso, 12, 44100 - Ferrara, Italy \\ and \\ ITEP, Bol. Cheremushkinskaya 25, Moscow 113259, Russia.
}

\begin{abstract}
The lectures describe several cosmological effects produced by neutrinos. Upper and lower cosmological limits on neutrino mass are derived. The role that neutrinos may play in formation of large scale structure of the universe is described and neutrino mass limits are presented. Effects of neutrinos on cosmological background radiation and on big bang nucleosynthesis are discussed. Limits on the number of neutrino flavors and mass/mixing are given.
\end{abstract}

\section{Introduction}

Of all known particles neutrinos have the weakest interactions and the smallest possibly nonvanishing, mass. Thanks to these properties neutrino is the second most abundant particle in the universe after photons. According to observations the number density of photons in cosmic microwave background radiation (CMBR) is $n_{\gamma}=412 / \mathrm{cm}^{3}$. In standard cosmology the number density of cosmic neutrinos can be expressed through $n_{\gamma}$ as

$$
n_{\nu}+n_{\bar{\nu}}=3 n_{\gamma} / 11=112 / \mathrm{cm}^{3}
$$

for any neutrino flavor $\left(\nu_{e}, \nu_{\mu}\right.$, and $\left.\nu_{\tau}\right)$, assuming that there is an equal number of neutrinos and antineutrinos.

Knowing the temperature of CMBR, $T_{\gamma}=2.728 \mathrm{~K}=2.35 \cdot 10^{-4} \mathrm{eV}$, one can calculate the temperature of cosmic neutrinos:

$$
T_{\nu}=(4 / 11)^{1 / 3} T_{\gamma}=1.95 \mathrm{~K}=1.68 \cdot 10^{-4} \mathrm{eV}
$$


which is true if the neutrino mass is much smaller than their temperature, $m_{\nu} \ll T_{\nu}$. Otherwise the parameter $T_{\nu}$ does not have the meaning of temperature; up to a constant factor it can be understood as the inverse cosmological scale factor $a(t)$. Theory predicts that the spectrum of cosmic neutrinos, even massive ones, is given by the almost equilibrium form:

$$
f_{\nu}=[\exp (p / T-\xi)+1]^{-1}
$$

with the dimensionless chemical potential $\xi=\mu / T$ usually assumed to be negligibly small. However one should note that in the expression above $p$ is the neutrino momentum, while in the equilibrium distribution there stands energy $E=\sqrt{m_{\nu}^{2}+p^{2}}$.

There is a small correction to expression (3) of the order of $\left(m_{\nu} / T_{d}\right)^{2}$ where $T_{d}$ is the neutrino decoupling temperature, $T_{d} \sim \mathrm{MeV}$ - this is the temperature when neutrinos stopped to interact with primeval plasma. This correction appeared because at $T>T_{d}$ neutrinos were in equilibrium and their distribution depended on $E / T$. Distribution of noninteracting neutrinos should be a function of $p a(t)$. In the case of instantaneous decoupling it turns into $f\left(\sqrt{(p / T)^{2}+\left(m_{\nu} / T_{d}\right)^{2}}\right)$, while for non-instantaneous decoupling the dependence on mass could be different.

More details about cosmological neutrinos can be found e.g. in a recent review paper [1].

Neutrinos are normally assumed to possess only usual weak interactions with $(V-A)$-coupling to $W$ and $Z$ bosons. Correspondingly, if $m_{\nu}=0$, only left-handed neutrinos, $\nu_{L}$, i.e. those with spin anti-parallel to their momentum (and parallel for $\bar{\nu}$ ), possess this interaction, while right-handed neutrinos, $\nu_{R}$, are sterile. If $m_{\nu} \neq 0$ righthanded neutrinos would be also coupled to intermediate bosons with the strength suppressed as $\left(m_{\nu} / E\right)^{2}$ and their cosmological number density would be always negligible since their mass is bounded from above by a few eV see eqs. (13,14). If however neutrinos are mixed and massive (possibly with Majorana and Dirac masses) additional 
three sterile neutrinos could be abundantly produced in the early universe [2].

It is known from experiment that there are at least three neutrino families (or flavors), $\nu_{e}, \nu_{\mu}$, and $\nu_{\tau}$. From LEP data the number of light neutrino flavors with $m_{\nu}<m_{Z} / 2$ is indeed three:

$$
N_{\nu}=2.993 \pm 0.011
$$

One can find references to original experimental papers in the Review of Particle Physics [3].

Direct experiment limits on neutrino masses are [3]

$$
m_{\nu_{e}}<3 \mathrm{eV}, m_{\nu_{\mu}}<190 \mathrm{keV}, m_{\nu_{\tau}}<18.2 \mathrm{MeV}
$$

As we will see below, cosmology allows to derive an upper limit on masses of all neutrino flavors similar to that presented above for $m_{\nu_{e}}$.

There is a strong evidence in favor of neutrino oscillations. The best fit solutions to the observed neutrino anomalies indicates maximum mixing between $\nu_{\mu}$ and $\nu_{\tau}$ with mass difference about $3 \times 10^{-3} \mathrm{eV}^{2}$ (for explanation of atmospheric anomaly) and also large mixing between $\nu_{e}$ and another active neutrino with mass difference between $10^{-3}-10^{-5} \mathrm{eV}^{2}$ (for explanation of the deficit of solar neutrinos). If mass differences are indeed so small then masses of all active neutrinos should be below $3 \mathrm{eV}$ and right-handed neutrinos would not be practically produced in particle interactions in the standard theory, but as we noted below, they may be produced by oscillations.

Except for the above mentioned anomalies, and possibly LSND, neutrinos are well described by the standard electroweak theory. For a recent review of neutrino anomalies see e.g. ref. [4].

In what follows we discuss the bounds in neutrino masses that can be derived from the magnitude of cosmic energy density and large scale structure of the universe (sec. 1). Relation between cosmological neutrinos and CMBR is considered in sec. 
田. In section 5 we describe the role played by neutrinos in big bang nucleosynthesis $(\mathrm{BBN})$ and present the limits on the number of neutrino species and possible neutrino degeneracy. Cosmological impact of neutrino oscillations is considered in sec. 6. The body of the lectures is preceded by a brief presentation of basic cosmological facts and essential observational data (section Q). These lectures present a shorter version of the recent review paper [1] where one can find details and a long list or references, many of which are omitted here because of lack of space and time.

\section{A little about cosmology}

The universe is known to expand according to the Hubble law:

$$
V=H r
$$

where $V$ is the velocity of a distant object, $r$ is the distance to it and $H=\dot{a} / a$ is the Hubble constant (or better to say, Hubble parameter, since it is not constant in time). The present day value of $H$ is given by

$$
H=100 h \mathrm{~km} / \mathrm{sec} / \mathrm{Mpc}
$$

with $h=0.7 \pm 0.1$. There are still indications for smaller and larger values of $H$ but we will not go into details here. One can find discussion of determination and values of this and other cosmological parameters e.g. in recent papers [5].

The critical or closure energy density is proportional to $H^{2}$ and is equal to:

$$
\rho_{c}=\frac{3 H^{2} m_{P l}^{2}}{8 \pi}=10.6 h^{2} \mathrm{keV} / \mathrm{cm}^{3}
$$

where $m_{P l}=1.221 \mathrm{GeV}$ is the Planck mass. Contributions of different forms of matter into cosmological energy density is usually presented in terms of dimensionless parameter $\Omega_{j}=\rho_{j} / \rho_{c}$. According to the data the dominant part of cosmological energy density is given either by vacuum energy or by an unknown form of matter 
which has negative pressure, $p<-\rho / 3$, and induces an accelerated expansion (antigravity) at the present epoch. Its energy density is $\Omega_{v a c} \approx 0.7$. The total energy density is close to the critical value so $\Omega_{t o t} \approx 1$. The contribution of the usual baryonic matter, as determined from CMBR, is roughly $\Omega_{b} h^{2}=0.022$. This is is consistent with determination of $\Omega_{b}$ from BBN. The remaining 0.25 is believed to be contributed by some unknown elementary particles (though, say, black holes are not excluded) weakly interacting with photons - that's why they are called dark or invisible matter.

We are interested in a rather late period of the universe evolution when the temperature was in $\mathrm{MeV}$ range or below down to the present time. For more details about cosmology one can see any textbook or e.g. the recent reviews [1, 3]. Initially all the particles in primary plasma, photons, $e^{+} e^{-}$-pairs, three flavors of neutrinos and antineutrinos, and a little baryons were in strong thermal contact and hence had equilibrium distributions (3) for fermions and similar expressions with minus sign in front of 1 for bosons. Particle energy $E$ should stand in this equation instead of $p$ but since majority of particles are relativistic, this difference is not important. The energy density of massless particles in thermal equilibrium is given by the expression:

$$
\rho=\pi^{2} g_{*} T^{4} / 30
$$

where $g_{*}=10.75$ includes contribution from all mentioned above particles except for neglected baryons.

At that stage the energy density was almost precisely equal to the critical one, with accuracy better than $10^{-15}$, the particles were relativistic (for $T>m_{e}$ ) with equation of state $p=\rho / 3$, where $p$ is the pressure density, and thus:

$$
H=\frac{1}{2 t}=\left(\frac{8 \pi^{3} g_{*}}{90}\right)^{1 / 2} \frac{T^{2}}{m_{P l}}=5.443\left(\frac{g_{*}}{10.75}\right)^{1 / 2} \frac{T^{2}}{m_{P l}}
$$

Cross-section of neutrino interactions behaves as $\sigma_{\nu} \sim G_{F}^{2} E^{2}$ and the reaction rate is $\dot{n} / n \sim \sigma_{\nu} n \sim G_{F}^{2} T^{5}$. Here $n \sim T^{3}$ is the particle number density and $G_{F}=$ 
$1.166 \cdot 10^{-5} \mathrm{GeV}^{2}$ is the Fermi coupling constant. Comparing reaction rate with the Hubble expansion rate we can conclude that at temperatures above a few $\mathrm{MeV}$ neutrinos should be in thermal equilibrium. To be more precise one should consider kinetic equation governing neutrino distribution in cosmological background:

$$
\left(\partial_{t}+H p \partial_{p}\right) f_{\nu}(t, p)=H x \partial_{x} f_{\nu}(x, y)=I_{\text {coll }}
$$

where $p$ is the neutrino momentum, $x=1 / a(t), y=p a / m_{0}, a(t)$ is the cosmological scale factor, and $m_{0}$ is the normalization mass which we take as $m_{0}=1 \mathrm{MeV}$. On relativistic stage when $T \sim 1 / a$ is convenient to take $x=m_{0} / T$ and $y=p / T$.

For an estimate of neutrino decoupling temperature we neglect inverse reactions in the collision integral and assume Boltzmann statistics. Each of the neglected effects would enlarge the decoupling temperature by about 10-15\%. In this approximation the kinetic equation becomes

$$
H x \frac{\partial f_{\nu}}{f_{\nu} \partial x}=-\frac{D G_{F}^{2} y}{3 \pi^{3} x^{5}}
$$

where $D$ is a constant. Usually in the estimates of decoupling temperature one takes thermally average value of neutrino momentum, $\langle y\rangle=3$. If we include all possible reactions where neutrinos may participate then $D=80\left(1+g_{L}^{2}+g_{R}^{2}\right)$ with $g_{L}=\sin ^{2} \theta_{W} \pm 1 / 2$ and $g_{R}=\sin ^{2} \theta_{W}$ where $\sin ^{2} \theta_{W}=0.23$ and the sign " + " stands for $\nu_{e}$ and " ${ }^{\prime \prime}$ stands for $\nu_{\mu, \tau}$. Correspondingly the decoupling temperature determined with respect to the total reaction rate would be $T_{\nu_{e}}^{d}=1.34 \mathrm{MeV}$ and $T_{\nu_{\mu}, \nu_{\tau}}^{d}=1.5$ $\mathrm{MeV}$ for $\nu_{e}$ and $\nu_{\mu, \tau}$ respectively.

If we take into account only $(\nu-e)$-interactions then $D=80\left(g_{L}^{2}+g_{R}^{2}\right)$ and the decoupling of neutrinos from electron-positron (and photon) plasma would take place at $T_{\nu_{e}}^{d}=1.87 \mathrm{MeV}$ and $T_{\nu_{\mu}, \nu_{\tau}}^{d}=3.12 \mathrm{MeV}$. Above $T^{d}$ the temperatures of photonelectron-positron and neutrino plasma should be equal. In fact in the standard model equality is maintained down to $T \approx m_{e}$ (see below). 
If only $\nu \bar{\nu}$-annihilation into $e^{+} e^{-}$is taken into account then $D=16\left(g_{L}^{2}+g_{R}^{2}\right)$ and the decoupling temperatures would be $T_{\nu_{e}}^{d}=3.2 \mathrm{MeV}$ and $T_{\nu_{\mu}, \nu_{\tau}}^{d}=5.34 \mathrm{MeV}$. Below these temperatures the total number density of neutrinos in comoving volume could not change.

When temperature dropped below electron mass, $e^{+} e^{-}$-pairs annihilated heating photons but leaving neutrinos intact. As a result of this heating $T_{\gamma}$ become higher than $T_{\nu}$, eq. (2), and relative neutrino number density dropped with respect to photons as given by eq. (11) instead of earlier existed equilibrium ratio $\left(n_{\nu}+n_{\bar{\nu}}\right) / n_{\gamma}=3 / 4$.

\section{Cosmological limits on neutrino mass H $^{\prime}$}

\subsection{Gerstein-Zeldovich limit}

Since the number density of neutrinos at the present day is known, see eq. (11), it is easy to calculate their contribution into cosmological energy density, $\rho_{\nu}=\sum m_{\nu_{a}} n_{\nu}$, if neutrinos are stable. Demanding that $\rho_{\nu}$ does not exceed the known value of energy density of matter we obtain

$$
\sum_{a} m_{\nu_{a}}<95 \mathrm{eV} \Omega_{m} h^{2} \approx 14 \mathrm{eV}
$$

where the sum is taken over all light neutrino species, $a=e, \mu, \tau$. This limit was originally derived by Gerstein and Zeldovich [0] in 1966. Six years later the result was rediscovered by Cowsik and McClelland [8]. In the later paper, however, the photon heating by $e^{+} e^{-}$-annihilation was not taken into account and both helicity states of massive neutrinos were assumed to be equally abundant. Correspondingly the resulting number density of relic neutrinos was overestimated by the factor $11 / 2$.

If all active neutrinos are strongly mixed and their mass differences are very small (see the end of sec. 1) then the limit (13) for an individual mass would be $m_{\nu}<4.7$ $\mathrm{eV}$.

\footnotetext{
${ }^{1}$ More detailed discussion of such limits can be found in the recent reviews [1], 6].
} 
The bound (13) can be noticeably strengthened because neutrino may make only sub-dominant contribution to $\Omega_{m}$. Arguments based on large scale structure formation (see below sec. 3.3) lead to the conclusion that $\Omega_{\nu}<\Omega_{m} / 3$ and correspondingly:

$$
\sum_{a} m_{\nu_{a}}<5 \mathrm{eV}
$$

As noted above, in the case of small mass differences the mass bound for a single neutrino would be $m_{\nu}<1.7 \mathrm{eV}$.

\subsection{Tremaine-Gunn limit}

Quantum mechanics allows to obtain a lower limit on neutrino mass if neutrinos make all dark matter in galaxies, especially in dwarf ones [9]. The derivation is based on the fact that neutrinos are fermions and hence cannot have an arbitrary large number density if their energy is bounded from above to allow formation of gravitationally bound cluster. So to make dominant contribution into dark matter neutrino mass should be larger than a certain value. Gravitationally bound neutrinos would be most densely packed if they form degenerate gas with Fermi momentum $p_{f}=m_{\nu} V_{F}$. The Fermi velocity $V_{F}$ can be determined from the virial theorem:

$$
V_{F}^{2}=G_{N} M_{\text {gal }} / R_{\text {gal }}
$$

where $G_{N}=1 / m_{P l}^{2}$ is the Newton gravitational constant and $M_{g a l}$ and $R_{g a l}$ are respectively the mass and radius of a galaxy.

The number density of degenerate neutrinos and equal number of antineutrinos is $n_{\nu}=p_{F}^{3} /\left(3 \pi^{2}\right)$ and correspondingly their total mass in a galaxy is

$$
M_{\nu}=4 \pi R_{\text {gal }}^{3} m_{\nu} n_{\nu} / 3
$$

According to observations galactic masses are dominated by invisible matter, so one should expect that $M_{\nu} \approx M_{\text {gal }}$. From the equations above we find:

$$
m_{\nu}=80 \mathrm{eV}\left(\frac{300 \mathrm{~km} / \mathrm{sec}}{V}\right)^{1 / 4}\left(\frac{1 \mathrm{kpc}}{R_{\text {gal }}}\right)^{1 / 2}
$$


For dwarfs $R_{g a l} \approx 1 \mathrm{kpc}$ and $V \approx 100 \mathrm{~km} / \mathrm{sec}$. Correspondingly neutrinos, if they constitute all dark matter in such galaxies, should be rather heavy, $m_{\nu}>100 \mathrm{eV}$ in contradiction with Gerstein-Zeldovich limit. Thus we have to conclude that dark matter in galaxies is dominated by some other unknown particles.

\subsection{Neutrinos and large scale structure of the universe}

Though, as we saw above, massive neutrinos cannot be dominant dark matter particles, they may play an essential role in large scale structure formation and evolution. According to the accepted point of view cosmological structures have been developed as a result of gravitational instability of initially small primordial density perturbations. The latter presumably were generated at inflationary stage due to rising quantum fluctuations of the inflaton field. For reviews and list of references see e.g. [10]. It is usually assumed that the spectrum of initial density perturbation has a simple power law form, i.e. Fourier transform of the density perturbations

$$
(\delta \rho / \rho)_{i n}=\int d^{3} k \delta(k)
$$

behaves as $\delta^{2} \sim k^{n}$. Moreover, the value of the exponent, $n$, is usually taken to be 1 . It corresponds to flat or Harrison-Zeldovich spectrum [11], as indicated by inflation and consistent with observations.

With the known initial perturbations and equation of state of cosmological matter one can calculate the shape of the evolved spectrum and to compare it with observations. This permits to determine the properties of the cosmological dark matter. In the case of neutrinos density perturbations at small scales are efficiently erased as can be seen from the following simple arguments. Neutrinos were decoupled from plasma when they relativistic. The decoupling temperature is $T^{d} \sim \mathrm{MeV}$, while $m_{\nu} \leq 10$ $\mathrm{eV}$. Thus after decoupling neutrinos free streamed practically with the speed of light. Since the flux of neutrinos from neutrino-rich regions should be larger than that from 
neutrino-poor regions, the inhomogeneities in neutrino distribution would smoothed down at the scales smaller than neutrino free path, $l_{f s}=2 t_{n r}$. Here $t_{n r}$ is the cosmic time from beginning till the moment when neutrinos became nonrelativistic. As we mentioned above neutrinos propagate with the speed of light, so locally their path is equal just to $t$ and factor 2 came from the expansion of the universe. The mass contained inside $l_{f s}$ is

$$
M_{f s}=\frac{4 \pi\left(2 t_{f s}\right)^{3}}{3} \rho=m_{P l}^{2} t_{f s}
$$

where we used for the cosmological energy density the critical value (8) with the Hubble parameter $H=1 /(2 t)$, as given by eq. (10). Assuming that the universe was dominated by relativistic matter (photons and three neutrino flavors) till neutrino temperature dropped down to $T_{\nu}=m_{\nu} / 3$ and taking into account that $T_{\nu} \approx 0.7 T_{\gamma}$ (2) we find that the mass inside the free-streaming length is

$$
M_{f s}=0.1 m_{P l}^{3} / m_{\nu}^{2} \approx 10^{17} M_{\odot}\left(\mathrm{eV} / m_{\nu}\right)^{2}
$$

where $M_{\odot}=2 \cdot 10^{33} \mathrm{~g}$ is the solar mass. This result is derived for the case of one neutrino much heavier than the others. It would be modified in an evident way if neutrinos are mass degenerate.

In such a theory the characteristic mass of the first formed objects, $M_{f s}$, is much larger than the mass of large galaxies, $M_{\text {gal }} \sim 10^{12} M_{\odot}$ and dark matter with such property is called hot dark matter (HDM). The dark matter particles for which the characteristic mass is smaller than the galactic mass are called cold dark matter $(\mathrm{CDM})$ and the intermediate case is naturally called warm (WDM). In HDM model of structure formation large clusters of galaxies should be formed first and smaller structures could be created from larger ones later by their fragmentation. However such process demands too much time and, moreover, the observations indicate that smaller structures are older. Together with Tremaine-Gunn limit discussed in sec. 3.2, 
it "twice" excludes neutrinos as dominant part of dark matter in the universe. However mixed models with comparable amount of CDM and HDM are not excluded. Though the mystery of cosmic conspiracy - why different particles have comparable contribution to $\Omega$ - becomes in this case even more pronounced:

$$
\Omega_{v a c} \sim\left(\Omega_{m}=\Omega_{C D M}+\Omega_{H D M}\right) \sim \Omega_{b}
$$

From the arguments presented here one can see that the larger is the fraction of neutrinos in the total mass density of the universe the smaller should be power in cosmic structures at small scales. This permits to strengthen the upper limit on neutrino mass. Especially sensitive to neutrino mass are the structures at large red-shift $z$ because in neutrino dominated universe small structures should form late and should not exist at large $z$. The neutrino impact on the structure formation was analyzed in refs. [12 with the typical limits between 1 and $5 \mathrm{eV}$. More detailed discussion and more references can be found in the review [1]. According to ref. [13] Sloan Digital Sky Survey is potentially sensitive to $m_{\nu} \leq 0.1 \mathrm{eV}$.

\subsection{Cosmological limit on heavy neutrino mass}

If there exists fourth lepton generation then the corresponding neutrino should be heavier than $m_{Z} / 2=45 \mathrm{GeV}$ to surpass the LEP result (田). If these heavy neutrinos are stable on cosmological time scale, $\tau_{\nu} \geq t_{U} \sim 10^{10}$ years, then their mass density may be cosmologically noticeable. Since such neutrinos are assumed to be very heavy their number density at decoupling should be Boltzmann suppressed and they may escape Gerstein-Zeldovich limit. First calculations of cosmological number density of massive particles were performed by Zeldovich in 1965 [14]. However his result contained a numerical error later corrected in ref. [15. The same approach was applied to the calculations of the number/energy density of relic heavy neutrinos

practically simultaneously in the papers [16] where it was found that the mass of 
heavy neutrino should be above $2.5 \mathrm{GeV}$ to be cosmologically safe.

The number density of massive particle (neutrinos)s which survived annihilation is inversely proportional to the annihilation cross-section $\sigma_{a n n}$ and is approximately given by the expression

$$
n_{\nu} / n_{\gamma}=\left(\sigma_{a n n} v m_{\nu} m_{P l}\right)^{-1}
$$

where $v$ is the c.m. velocity of the annihilating particles and $n_{\gamma}$ is the number density of photons in CMBR. For relatively light neutrinos, $m_{\nu} \ll m_{Z}$ (which is not realistic now), the annihilation cross-section is proportional to $\sigma \sim m_{\nu}^{2}$ and the energy density of heavy relic neutrinos drops as $1 / m_{\nu}^{2}$. According to the calculations quoted above $\rho_{\nu}=\rho_{c}$ for $m_{\nu}=2.5 \mathrm{GeV}$.

For higher masses $m_{\nu}>m_{W, Z}$, the cross-section started to drop as $\sigma \sim \alpha^{2} / m_{\nu}^{2}$ and the cosmologically allowed window above $2.5 \mathrm{GeV}$ becomes closed for $m_{\nu}>(3-5)$ $\mathrm{TeV}$ [17]. However for $m_{\nu}>m_{W}$ a new channel of annihilation becomes open,

$$
\nu_{h}+\bar{\nu}_{h} \rightarrow W^{+} W^{-}
$$

with the cross-section rising as $m_{\nu_{h}}$ [18]. The rise of the cross-section is related to the rise of the Yukawa couplings of Higgs boson which is necessary to ensure a large mass of $\nu_{h}$. Correspondingly the excluded region above a few $\mathrm{TeV}$ becomes open again. However the annihilation (23) proceeds only in one lowest partial wave and the cross-section is restricted by the unitarity limit [19],

$$
\sigma_{J}<\pi(2 J+1) / p^{2}
$$

If one assumes that this limit is saturated then the large values $m_{\nu_{h}}$ about $100 \mathrm{TeV}$ would be forbidden. In reality the limit should be somewhat more restrictive because it is natural to expect that the cross-section started to drop with rising mass of neutrino before it reaches the unitarity bound. However it is very difficult, if possible at all, to make any accurate calculations in this strong interaction regime. 
To summarize this discussion, the cosmic energy density, $\rho_{\nu_{h}}$, of heavy neutrinos with the usual weak interaction is sketched in fig. (1). In the region of very small masses the ratio of number densities $n_{\nu_{h}} / n_{\gamma}$ does not depend upon the neutrino mass and $\rho_{\nu_{h}}$ linearly rises with mass. For larger masses $\sigma_{a n n} \sim m_{\nu_{h}}^{2}$ and $\rho_{\nu_{h}} \sim 1 / m_{\nu_{h}}^{2}$. This formally opens a window for $m_{\nu_{h}}$ above $2.5 \mathrm{GeV}$. A very deep minimum in $\rho_{\nu_{h}}$ near $m_{\nu_{h}}=m_{Z} / 2$ is related to the resonance enhanced cross-section around $Z$-pole. Above $Z$-pole the cross-section of $\bar{\nu}_{h} \nu_{h}$-annihilation into light fermions goes down with mass as $\alpha^{2} / m_{\nu_{h}}^{2}$ (as in any normal weakly coupled gauge theory). The corresponding rise in $\rho_{\nu_{h}}$ is shown by a dashed line. However for $m_{\nu_{h}}>m_{W}$ the contribution of the channel $\bar{\nu}_{h} \nu_{h} \rightarrow W^{+} W^{-}$leads to the rise of the cross-section with increasing neutrino mass as $\sigma_{a n n} \sim \alpha^{2} m_{\nu_{h}}^{2} / m_{W}^{4}$. This would allow keeping $\rho_{\nu_{h}}$ well below $\rho_{c}$ for all masses above $2.5 \mathrm{GeV}$. The behavior of $\rho_{\nu_{h}}$, with this effect of rising cross-section included, is shown by the solid line up to $m_{\nu_{h}}=1.5 \mathrm{TeV}$. Above that value it continues as a dashed line. This rise with mass would break unitarity limit for partial wave amplitude when $m_{\nu_{h}}$ reaches $1.5 \mathrm{TeV}$ (or $3 \mathrm{TeV}$ for Majorana neutrino). If one takes the maximum value of the S-wave cross-section permitted by unitarity (24), which scales as $1 / m_{\nu_{h}}^{2}$, this would give rise to $\rho_{\nu_{h}} \sim m_{\nu_{h}}^{2}$ and it crosses $\rho_{c}$ at $m_{\nu_{h}} \approx 200 \mathrm{TeV}$. This behavior is continued by the solid line above $1.5 \mathrm{TeV}$. However for $m_{\nu_{h}} \geq$ a few $\mathrm{TeV}$ the Yukawa coupling of $\nu_{h}$ to the Higgs field becomes strong and no reliable calculations of the annihilation cross-section has been done in this limit. Presumably the cross-section is much smaller than the perturbative result and the cosmological bound for $m_{\nu_{h}}$ is close to several $\mathrm{TeV}$. This possible, though not certain, behavior is presented by the dashed-dotted line. One should keep in mind, however, that the presented results for the energy density could only be true if the temperature of the universe at an early stage was higher than the heavy lepton mass. 


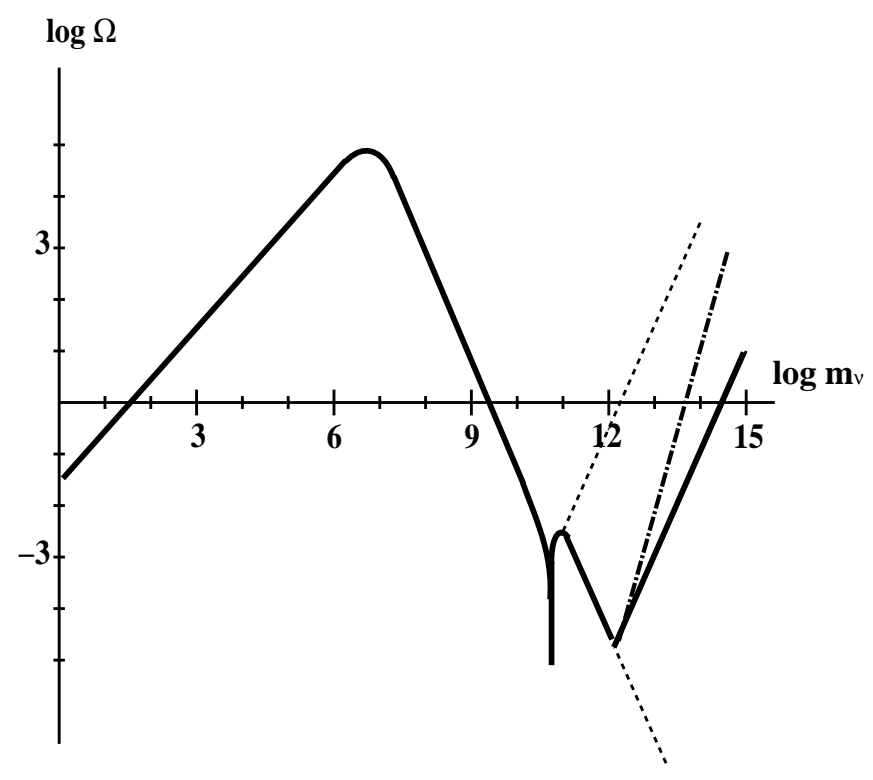

Figure 1: Cosmological energy density of massive neutrinos $\Omega=\rho_{\nu_{h}} / \rho_{c}$ as a function of their mass measured in $\mathrm{eV}$. The meaning of different lines is explained in the text.

\section{Neutrinos and CMBR}

Measurements of the angular fluctuations of CMBR, which are in continuous progress now, also permit to obtain valuable information about cosmic neutrinos. The spectrum of fluctuations is presented in terms of $C_{l}$, the squares of the amplitudes in the decomposition of the temperature fluctuations in terms of spherical harmonics:

$$
\frac{\Delta T}{T}=\sum_{l, m} a_{l m} Y_{l m}(\theta, \phi)
$$

and

$$
C_{l}=\frac{1}{2 l+1} \sum_{m=-l}^{l}\left|a_{l m}\right|^{2}
$$

A typical spectrum of fluctuations is presented in fig. Z(a) [20]. 


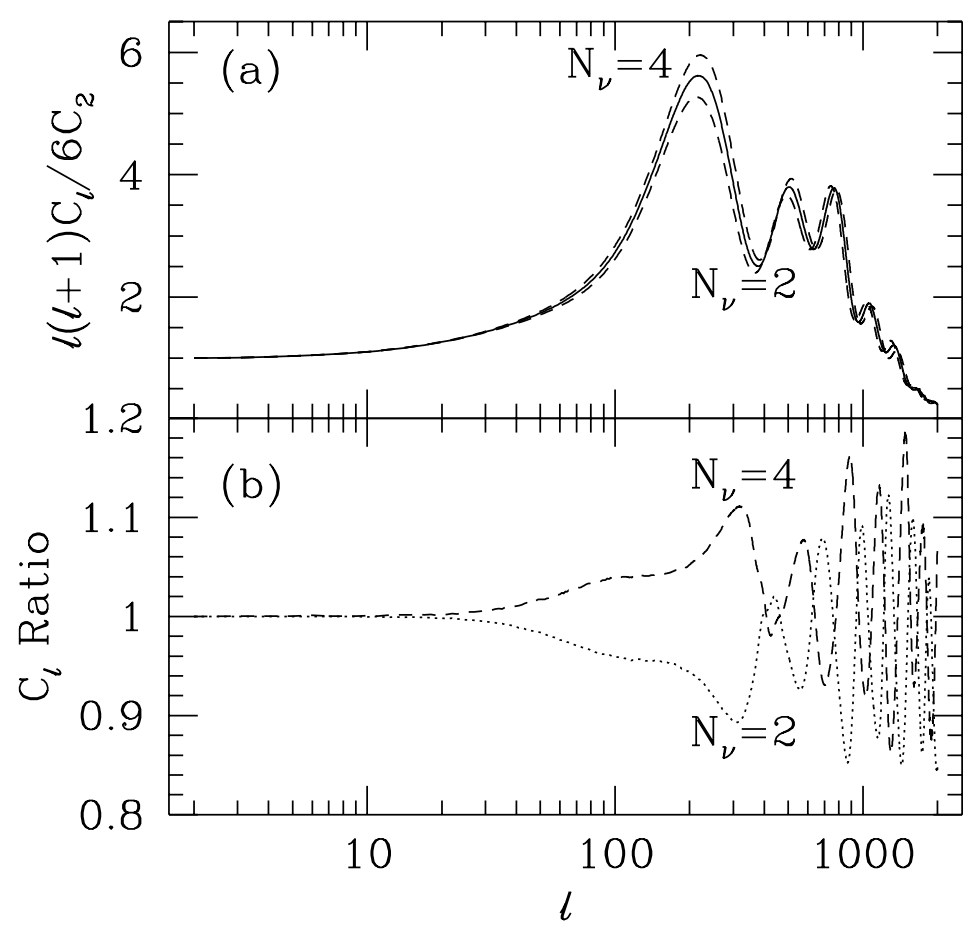

Figure 2: (a) An example of an angular spectrum of CMB anisotropies with varying number of neutrino species, $k_{\nu}=2,3$, 4 . (b) The ratio of $C_{l}$ for $k_{\nu}=2,4$ relative to $k_{\nu}=3$ (from ref. [20])

For low $l$ the amplitudes $C_{l}$ are practically $l$-independent if the spectrum of initial density perturbations is flat. At $l \approx 200$ it has a pronounced peak and a few weaker peaks at larger $l$. At $l>10^{3}$ the fluctuations are strongly damped. A detailed explanation of these spectral features can be found e.g. in the review [21]. These peaks were produced by sound waves at the earlier stage, roughly speaking at the moment of hydrogen recombination at $T \approx 3000 \mathrm{~K}$. After this moment the universe became transparent to CMB photons and the features existed at the moment of recombination became "frozen" and are observed now in the sky. The first peak of the largest amplitude corresponds to the "last" sound wave with the wave length 
equal to the horizon size at recombination divided by the speed of sound which for relativistic plasma is $c_{s}=1 / \sqrt{3}$ :

$$
\lambda_{1}=l_{h o r}^{(r e c)} / \sqrt{3}
$$

The other peaks correspond to maximum compression or rarefaction at the same moment and their phase is larger by $n \pi$. Their amplitude is typically smaller because corresponding waves was generated earlier and had more time to decay.

The physical scale $l_{h o r}^{(r e c)}$ depends upon the expansion regime and, in particular, upon the fraction of relativistic matter. Because of that the peak position is sensitive to number of neutrino families and to neutrino mass. However this effect is rather weak and to a much larger extent the position of the peak is determined by the geometry of the universe because the angle at which we see $l_{\text {hor }}^{(r e c)}$ at the present time depends upon the curvature of space. The data strongly support spatially flat universe, $\Omega_{t o t}=1$ (for an analysis of previous and new data see e.g. ref. 222).

More sensitive to the contribution of relativistic matter are heights of the peaks. The point is that at non-relativistic or, in other words, at matter-dominated (MD) stage gravitational potential of perturbations, $\psi$, remains constant. Indeed the potential satisfies the Laplace equation:

$$
\left(1 / a^{2}\right) \Delta \psi \sim \delta \rho
$$

where $a$ is the cosmological scale factor and $\delta \rho$ is the density contrast. It is known that density perturbations at MD-stage rise as $\delta \rho / \rho \sim a$, while the total energy density decreases as $\rho \sim 1 / a^{3}$. Correspondingly

$$
\psi \sim a^{2} \rho(\delta \rho / \rho)=\text { const }
$$

If the cosmological expansion is not exactly non-relativistic due to presence of some relativistic matter (neutrinos) the gravitational potential would be time depending, 
$\psi=\psi(t)$, and the sound waves (which are induced by gravity) would be amplified the effect is analogous to parametric resonance amplification.

The heights of the peaks also depend upon the fraction of baryonic matter, $\Omega_{b} h^{2}$ since the latter makes the main contribution to the mass of acoustic oscillators, while dark matter particles do not experience any pressure from photons of CMB. The degeneracy of the impact of different cosmological parameters on the angular spectrum of CMBR makes determination of these parameters much more difficult and one needs to invoke additional information from other pieces of astronomical data and/or to wait till more precise measurement of all $C_{l}$ in forthcoming experiments.

At the present day the accuracy of determination of the properties of cosmic neutrinos from CMBR is not very good. In ref. [23] the upper limit on the number of neutrino families was found $N_{\nu}<17$ (95\% confidence level) for the Hubble parameter $h=0.72 \pm 0.08$ and $\Omega_{b} h^{2}=0.020 \pm 0.002$. If these parameters are larger a larger fraction of relativistic energy density would be allowed and more neutrino flavors or other relativistic particles may exist. An additional account of the data on the large scale structure [23] permitted to arrive to an interesting lower limit, $N_{\nu}>1.5$. Thus an independent indication (in addition to BBN) of non-vanishing cosmological background of massless or very light neutrinos is obtained. Of course, these results

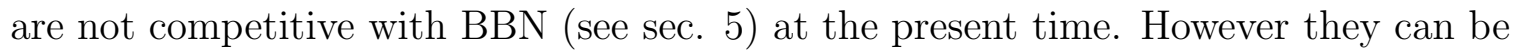
such in near future.

If neutrinos are massive and contribute into hot component of dark matter, their presence can be traced through CMBR [24]. Both effects mentioned above, a shift of the peak positions and a change of their heights, manifest themselves depending on the fraction of hot dark matter $\Omega_{H D M}$. Moreover the angular spectrum of CMBR is sensitive also to the value of neutrino mass because the latter shifts $t_{e q}$, the moment of the transition from radiation dominance to matter dominance. According to the paper [24] the amplitude of angular fluctuations of CMBR is 5-10\% larger for $400<$ 
$l<1000$ in the mixed hot-cold dark matter $(\mathrm{HCDM})$ model with $\Omega_{\nu}=0.2-0.3$ in comparison with the pure CDM model. An analysis of the data of ref. [25] on CMBR angular spectrum was performed in the paper. [26] and the best-fit range of neutrino mass was found:

$$
m_{\nu}=0.04-2.2 \mathrm{eV}
$$

An interesting effect which is related to neutrino physics in the early universe when the latter was about $1 \mathrm{sec}$ old and the temperature was in $\mathrm{MeV}$ range may be observed in the forthcoming Planck mission if the expected accuracy at per cent level is achieved. It is usually assumed that at that time neutrinos had an equilibrium spectrum with the temperature which was initially equal to the temperature of photons, electrons, and positrons, while somewhat later at $T<m_{e}$ neutrino temperature dropped with respect to the photon one because $e^{+} e^{-}$-annihilation heated photons but not neutrinos since neutrinos were already decoupled from electrons and positrons (see eq. (2) and discussion at the end of sec. 22). However the decoupling was not an instantaneous process and some residual interactions between $e^{ \pm}$and neutrinos still existed at smaller temperatures. The annihilation of the hotter electron-positron pairs, $e^{+} e^{-} \rightarrow \bar{\nu} \nu$, would heat up the neutrino component of the plasma and distort the neutrino spectrum. The average neutrino heating under assumption that their spectrum maintained equilibrium was estimated in ref. [27]. However, the approximation of the equilibrium spectrum is significantly violated and this assumption was abolished in subsequent works. In the earlier papers [28, 29] kinetic equations were approximately solved in Boltzmann approximation. In ref. [29] the effect was calculated numerically, while in ref. [28] an approximate analytical expression was derived. After correction of the numerical factor $1 / 2$ the calculated spectral distortion has the form:

$$
\frac{\delta f_{\nu_{e}}}{f_{\nu_{e}}} \approx 3 \cdot 10^{-4} \frac{E}{T}\left(\frac{11 E}{4 T}-3\right)
$$


Here $\delta f=f-f^{(e q)}$. The distortion of the spectra of $\nu_{\mu}$ and $\nu_{\tau}$ is approximately twice weaker.

An exact numerical treatment of the problem (i.e. numerical solution of the integro-differential kinetic equations without any simplifying approximations) was conducted in the papers [30]-32]. The accuracy of the calculations achieved in ref. [31] was the highest and some difference with the results of two other papers can be prescribed to a smaller number of grids in the collision integral [30] or to non-optimal distribution of them [32] (see discussion of different methods of calculations in ref. [1]). Recently calculations of the distortion of neutrino spectrum were done in ref. 333 in a completely different way using expansion in interpolating polynomials in momentum [34. The results of this work perfectly agree with those of ref. [31.

One would expect that the distortion of neutrino spectrum at a per cent level would result in a similar distortion in the primordial abundances of light elements. However, this does not occur because an excess of energetic neutrinos over the equilibrium spectrum which would give rise to a larger neutron-to-proton ratio and to a larger mass fraction of primordial ${ }^{4} \mathrm{He}$ is compensated by an increase of the total energy density of $\nu_{e}$ which acts in the opposite direction diminishing the neutron-proton freezing temperature and thus diminishing the $n / p$-ratio (see below sec. 5). The net result of this distortion on ${ }^{4} \mathrm{He}$ is at the level of $10^{-4}$. Still the observation of this small deviation of neutrinos from equilibrium is not impossible. The corrections discussed here and electromagnetic corrections of ref. [35] could be interpreted as a change of $N_{\nu}$ from 3 to 3.04. Planck mission may detect this effect but the concrete features depend upon the ratio of neutrino mass to the recombination temperature $T_{\text {rec }}=3000 \mathrm{~K}=0.26 \mathrm{eV}$.

\section{Neutrinos and BBN}




\subsection{BBN - brief description}

Physical processes essential for BBN took place when the temperature of the primeval plasma was in the interval from a few $\mathrm{MeV}$ down to $60-70 \mathrm{keV}$. According to eq. (10) the characteristic time was respectively between 0.1 sec up to 200 sec. During this period light elements ${ }^{2} \mathrm{H},{ }^{3} \mathrm{He},{ }^{4} \mathrm{He}$, and ${ }^{7} \mathrm{Li}$ were synthesized. As one can see from eq. (10) the universe cooling rate i.e. time-temperature relation depends on the number of particle species in the primeval plasma, $g_{*}$. At BBN epoch the latter is usually parameterized as

$$
g_{*}=10.75+(7 / 4)\left(N_{\nu}-3\right)
$$

where the effective number of additional neutrinos $\Delta N_{\nu}=N_{\nu}-3$ describes any form of energy present during BBN. This parameterization is precise if a non-standard energy has relativistic equation of state, $p=\rho / 3$. In any other case $\Delta N$ becomes a function of time and, moreover, the impact of additional energy on primordial abundances of different light elements could be different from that created by the equivalent number of massless neutrinos.

Building blocks for for creation of light elements were prepared in the weak interaction reactions:

$$
\begin{aligned}
n+\nu_{e} & \leftrightarrow p+e^{-}, \\
n+e^{+} & \leftrightarrow p+\bar{\nu}
\end{aligned}
$$

At high temperatures, $T>0.7 \mathrm{MeV}$, these reactions were fast in comparison with the universe expansion rate $H$ and the neutron-proton ratio followed the equilibrium curve:

$$
(n / p)=\exp (-\Delta m / T) \exp \left(-\xi_{\nu_{e}}\right)
$$

where $\Delta m=1.3 \mathrm{MeV}$ is the neutron-proton mass difference and $\xi_{\nu_{e}}=\mu_{\nu_{e}} / T$ is dimensionless chemical potential of electronic neutrinos. At smaller $T$ reactions (33, 34) 
became effectively frozen and the ratio $n / p$ would be constant if not the slow neutron decay with the life-time $\tau_{n}=885.7 \pm 0.8 \mathrm{sec}$ [3].

The temperature of the freezing of the reactions (33).34), $T_{n p}$, is determined by the competition of the reaction rate, $\Gamma \sim G_{F}^{2} T^{5}$ and the expansion rate, $H \sim \sqrt{g_{*}} T^{2}$. Hence $T_{n p} \sim g_{*}{ }^{1 / 6}$. Larger is $g_{*}$, larger is $T_{n p}$, and more neutrons would remain for creation of light elements. On the other hand, the nucleosynthesis temperature, $T_{N S}$, does not depend upon $g_{*}$ but the time when $T_{N S}$ is reached depends upon it. Larger is $g_{*}$ shorter is this time. Correspondingly less neutrons would decay and more helium-4 and deuterium would be created. Thus a variation of $g_{*}$ acts in the same direction in both phenomena. In particular, an increase of $N_{\nu}$ by 1 leads to an increase of ${ }^{4} \mathrm{He}$ by about $5 \%$ as one can easily check using the publicly available BBN code [36].

Light elements begin to form from the primeval protons and neutrons when the temperature reached the value

$$
T_{N S}=\frac{0.064 \mathrm{MeV}}{1-0.029 \ln \eta_{10}}
$$

where $\eta_{10}$ is the ratio of baryon and photon number densities in units $10^{10}$. A very small baryon-to-photon ratio makes nucleosynthesis temperature much lower than typical nuclear binding energies. A few years ago $\eta$ was determined from BBN itself and now the measurements of the angular spectrum of CMBR (see sec. 四) permit to find it independently. According to the latter $\eta_{10} \approx 5$. This result is in a reasonable agreement with determination of $\eta_{10}$ from BBN. Once $T_{N S}$ is reached light elements are very quickly formed and practically all neutrons, that survived to this moment, were binded in ${ }^{4} \mathrm{He}$. The mass fraction of this element was about $25 \%$, while relative number of deuterium nuclei (as well as ${ }^{3} \mathrm{He}$ ) with respect to hydrogen is (a few) $\times 10^{-5}$. Amount of ${ }^{7} \mathrm{Li}$ is about five orders of magnitude smaller. Primordial abundances of light elements as functions of $\eta_{10}$ are presented in fig. 通.

A slight increase of ${ }^{4} \mathrm{He}$ as a function of $\eta$ can be mostly explained by an increase of 

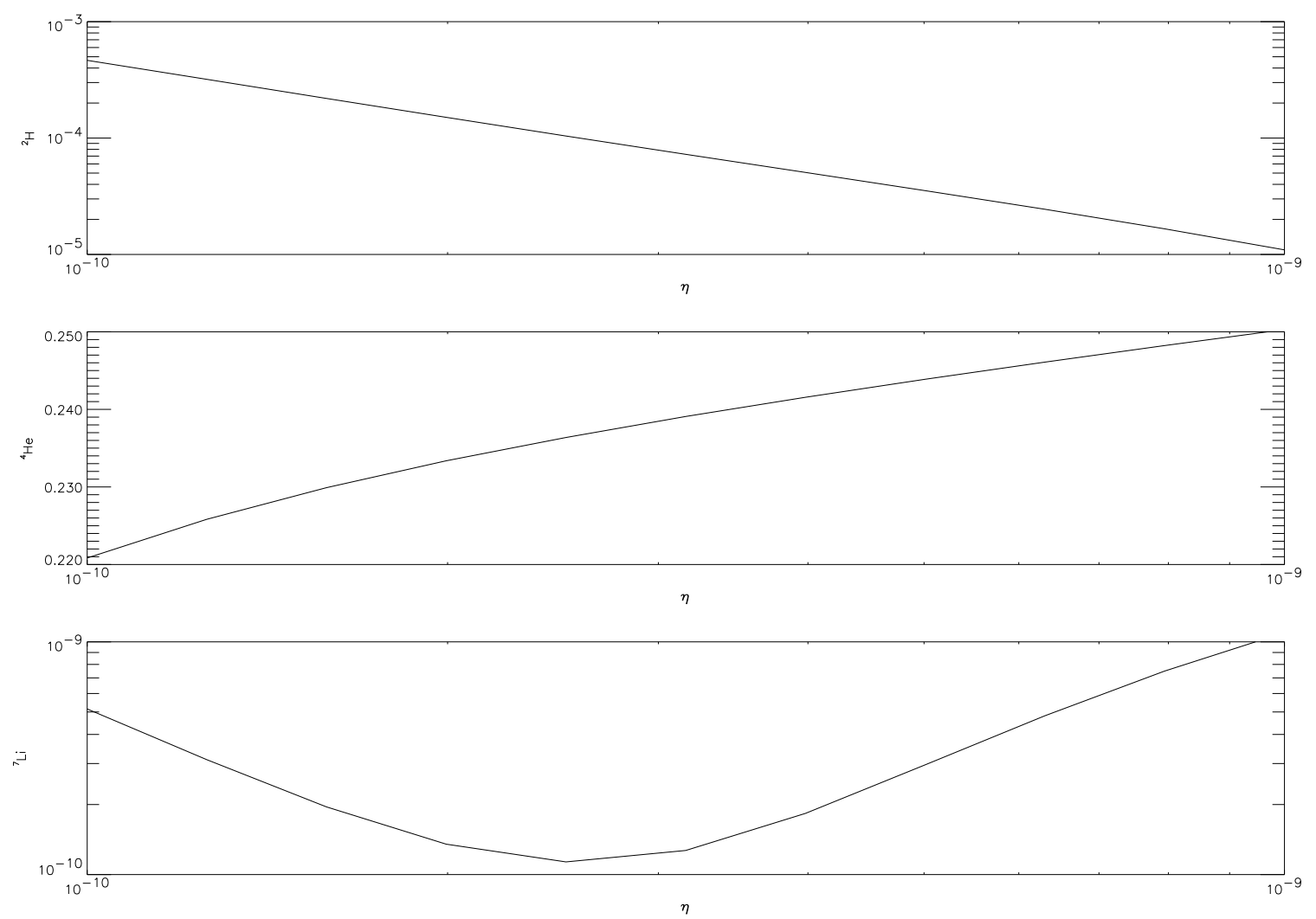

Figure 3: Abundances of light elements ${ }^{2} \mathrm{H}$ (by number) ${ }^{4} \mathrm{He}$ (by mass), and ${ }^{7} \mathrm{Li}$ (by number) as functions of baryon-to-photon ratio $\eta_{10} \equiv 10^{10} n_{B} / n_{\gamma}$.

the nucleosynthesis temperature (36) with rising $\eta$. Correspondingly nucleosynthesis started earlier and more neutrons survived decay. A strong decrease of the amount of the produced deuterium is explained by a larger probability for ${ }^{2} H$ to meet a nucleon and to proceed to ${ }^{4} \mathrm{He}$.

To summarize, we have seen that the primordial abundances depend upon:

1. Number density of baryons, $\eta=n_{B} / n_{\gamma}$.

2. Weak interaction rate; this is usually expressed in terms of the neutron lifetime. It is interesting that a variation of weak interaction strength by a factor 
of few would result either in complete absence of primordial ${ }^{4} \mathrm{He}$ or in $100 \%$ dominance of the latter (no hydrogen). In both cases stellar evolution would be quite different from what we observe.

3. Cosmological energy density; non-standard contribution is usually parameterized as additional number of neutrino species $\Delta N_{\nu}$.

4. Neutrino degeneracy; degeneracies of $\nu_{\mu}$ or $\nu_{\tau}$ are equivalent to a non-zero $\Delta N_{\nu}$, while degeneracy of $\nu_{e}$ has a much stronger (exponential) impact, see eq. (35), on the abundances because $\nu_{e}$ directly enters the (n-p)-transformation reactions (33,34) and can shift the $(n / p)$-ratio in either direction.

\subsection{Role of neutrinos in $\mathrm{BBN}$}

The role that neutrinos played in BBN is already clear from the previous section. The sensitivity of BBN to the number of neutrino families was first noticed by Hoyle and Tayler in 1964 [37]. Two years later a similar statement was made by Peebles [38]. More detailed calculations were performed by Shvartsman [39] in 1969 who explicitly stated that the data on light element abundances could be used to obtain a bound on the number of neutrino flavors. Another 8 years later Steigman, Schramm, and Gunn presented analysis of the effect with all light elements taken into account.

The dependence of the produced deuterium and helium- 4 on the number of neutrino species for different values of the baryon number density $\eta_{10}$ are presented in figs. 目, At the present day the conclusion about the allowed number of neutrino families varies from paper to paper between an optimistic constraint $\Delta N_{\nu}<0.2 \mathrm{up}$ to a more cautious one $\Delta N_{\nu}<1$. The review of these results can be found e.g. in refs. [41, 6].

If cosmological lepton asymmetry is non-vanishing then chemical potentials of neutrinos are non-zero and their energy density is higher than the energy density of 


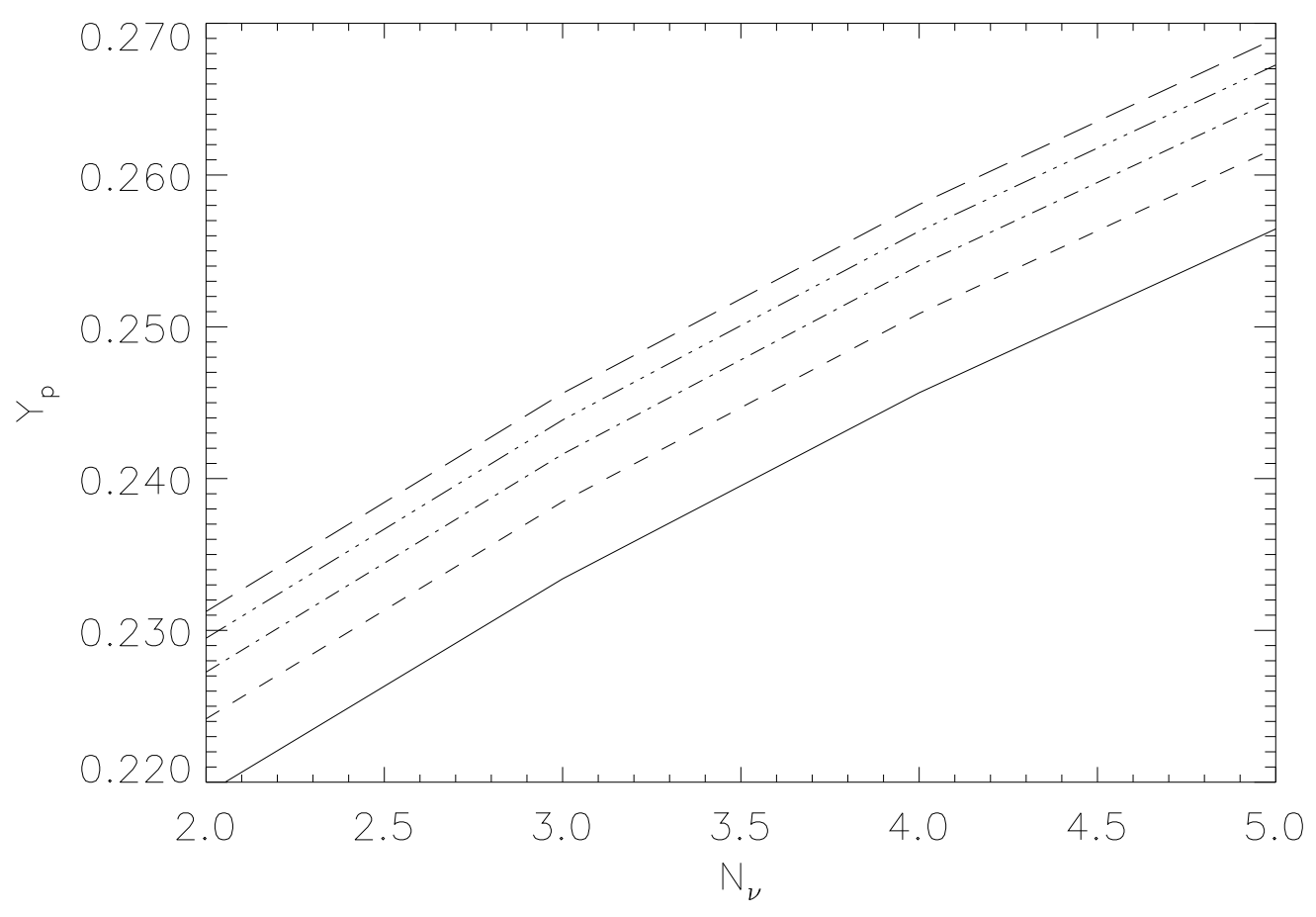

Figure 4: Mass fraction of ${ }^{4} \mathrm{He}$ as a function of the number of massless neutrino species. Different curves correspond to different values of the baryon-to-photon ratio $\eta_{10} \equiv 10^{10} n_{B} / n_{\gamma}=2,3,4,5,6$ in order of increasing helium abundance.

non-degenerate neutrinos. Additional effective number of neutrino species in this case is given by

$$
\Delta N(\xi)=\frac{15}{7} \sum_{a}\left[2\left(\frac{\xi_{a}}{\pi}\right)^{2}+\left(\frac{\xi_{a}}{\pi}\right)^{2}\right]
$$

where the sum is taken over all neutrino species $a=e, \mu, \tau$. If one extra family of neutrinos is allowed by BBN then chemical potentials of $\nu_{\mu}$ and $\nu_{\tau}$ are bounded by $\left|\xi_{\mu, \tau}\right|<1.5$. For electronic neutrinos the limit is much stronger, $\left|\xi_{e}\right|<0.1$ [42].

If combined variation of all chemical potentials is allowed such that by some conspiracy an increase in production of light elements due to non-zero $\xi_{\mu, \tau}$ is compensated 


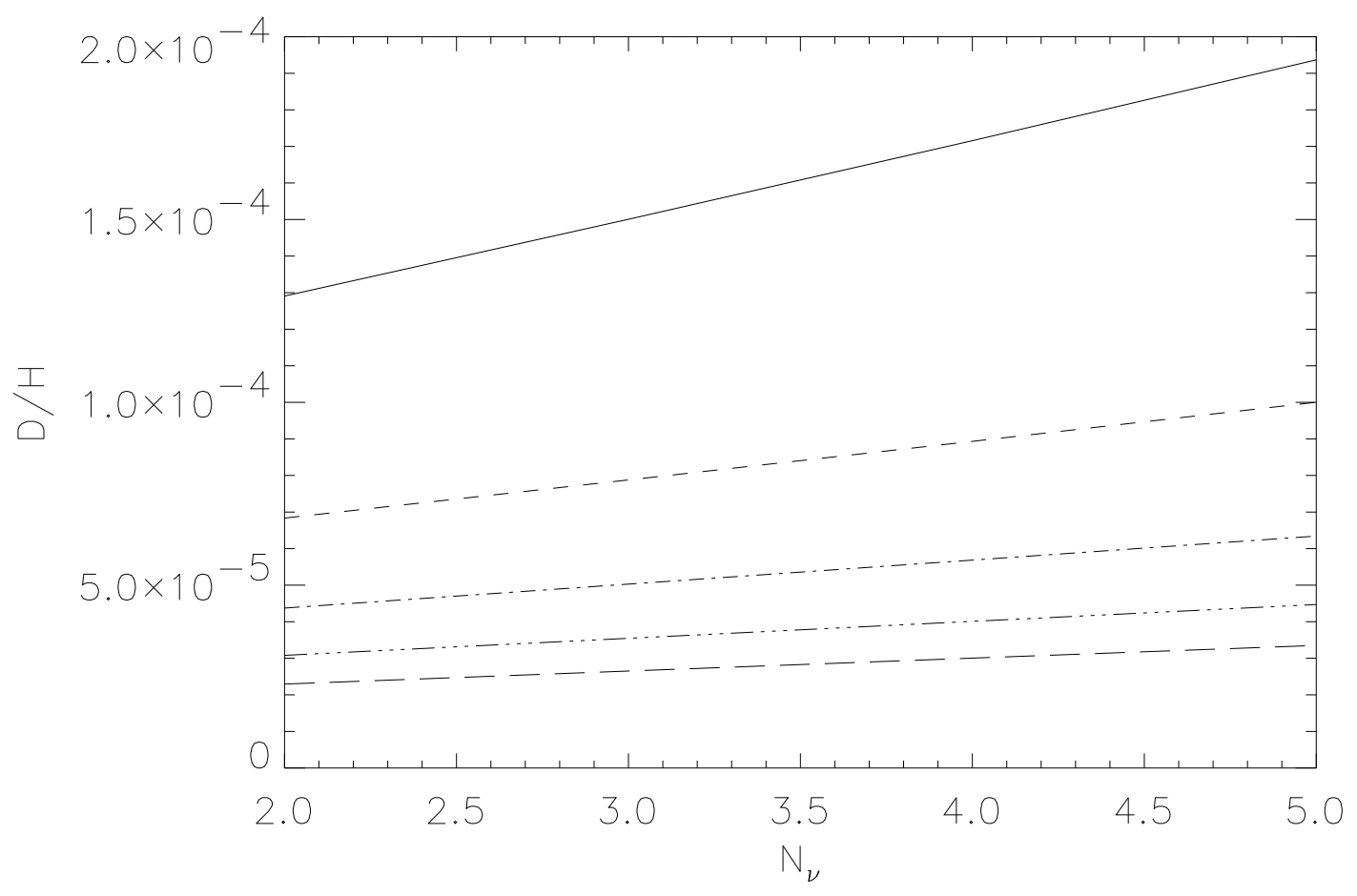

Figure 5: Deuterium-to-hydrogen by number as a function of the number of massless neutrino species. Notations are the same as in fig. (4).

by a positive $\xi_{e}$ then the limits would be less restrictive. Using additional data from CMBR which allowed to fix $\eta_{10}=5$ the authors of reference 43] obtained:

$$
\left|\xi_{\mu, \tau}\right|<2.6,-0.1<\xi_{e}<0.2
$$

This bound disregards mixing between active neutrinos discussed below in sec. 6.3 . Account of the latter may make this limit considerably more restrictive, eq. (64).

Distortion of spectrum of $\nu_{\mu}$ or $\nu_{\tau}$ would have an impact on primordial abundances only through a change in total energy density of these neutrinos and is equivalent to a change of $N_{\nu}$. However distortion of the spectrum of $\nu_{e}$ would have a stronger and non-monotonic influence on the abundances. If there is an increase of neutrinos in 
high energy tail of the spectrum then the frozen $n / p$-ratio would increase. Indeed an excess of $\nu_{e}$ in comparison with the equilibrium amount would lead to a more efficient destruction of neutrons in reaction (33), while the an excess of $\bar{\nu}$ would lead to more efficient production of neutrons in reaction (34). If spectral distortion is symmetric for particles and antiparticles then the second reaction would dominate because the number density of protons is 6-7 times larger than the number density of neutrons. It is worth noting that spectral distortion is not necessary charge symmetric as e.g. could be in the case of resonant oscillations between active and sterile neutrinos.

If spectrum of $\nu_{e}$ has an additional power at low energy then the $n / p$ ratio would decrease because the reaction (34), where neutrons are created, is suppressed at low energies due to threshold of $1.8 \mathrm{MeV}$.

If the spectrum is not distorted but the temperature of neutrinos is different from the temperature of photons then for a larger $T_{\nu_{e}}$ the $n / p$-ratio would be smaller because larger fraction of $\nu_{e}$ would shift the neutron freezing temperature, $T_{n p}$, to a smaller value. Thus an increase of the energy density of $\nu_{e}$ has an opposite effect on BBN than an increase of the energy density of $\nu_{\mu}$ or $\nu_{\tau}$.

\section{Neutrino oscillations in the early universe}

\subsection{Effects of medium}

There are serious reasons to believe that the mass eigenstates of neutrinos $\nu_{j}(j=$ $1,2, \ldots)$ do not coincide with interaction eigenstates, $\nu_{a}(a=e, \mu, \tau, \ldots)$. This mismatch leads to oscillation phenomenon. The interaction and mass eigenstates are expressed through each other by an unitary mixing matrix which in a simple case of two particle mixing (e.g. $\nu_{e}$ and $\nu_{\mu}$ ) has the form:

$$
\begin{aligned}
& \nu_{e}=\nu_{1} \cos \theta+\nu_{2} \sin \theta \\
& \nu_{\mu}=-\nu_{1} \sin \theta+\nu_{2} \cos \theta
\end{aligned}
$$


If a certain neutrino flavor (interaction eigenstate) is produced in space point $a$ then the probability to observe such state in space point $b$ oscillates as $\cos \left[(a-b) \delta m^{2} / 2 p\right]$ where $\delta m^{2}=m_{2}^{2}-m_{1}^{2}$ is the mass difference squared of the mass eigenstates.

Oscillations in matter are modified by neutrino effective potential $V_{\text {eff }}$ which up to energy factor coincides with refraction index of neutrinos in medium. The Schroedinger equation for neutrino vector wave function in medium can be written as

$$
i \partial_{t} \Psi=\left(\mathcal{H}_{m}+V_{\text {eff }}\right) \Psi
$$

where the matrix $\mathcal{H}_{m}$ is the free Hamiltonian which is diagonal in mass eigenstate basis, while effective potential, $V_{\text {eff }}$, is often (but not always, see below, sec. 6.3) diagonal in interaction eigenstate basis.

Diagonal entries of $V_{\text {eff }}$ for an active neutrino were calculated in ref. [44]:

$$
V_{e f f}^{(a a)}= \pm C_{1} \eta^{(a)} G_{F} T^{3}+C_{2}^{a} \frac{G_{F}^{2} T^{4} E}{\alpha}
$$

where $E$ is the neutrino energy, $T$ is the plasma temperature, $G_{F}$ is the Fermi coupling constant, $\alpha=1 / 137$ is the fine structure constant, and the signs " \pm " refer to antineutrinos and neutrinos respectively According to ref. [44] the coefficients $C_{j}$ are: $C_{1} \approx 0.95, C_{2}^{e} \approx 0.61$ and $C_{2}^{\mu, \tau} \approx 0.17$ (for $T<m_{\mu}$ ). These values are true in the limit of thermal equilibrium, otherwise these coefficients are some integrals from the distribution functions over momenta. The charge asymmetry of plasma is described by the coefficients $\eta^{(a)}$ which are equal to

$$
\begin{array}{r}
\eta^{(e)}=2 \eta_{\nu_{e}}+\eta_{\nu_{\mu}}+\eta_{\nu_{\tau}}+\eta_{e}-\eta_{n} / 2\left(\text { for } \nu_{e}\right) \\
\eta^{(\mu)}=2 \eta_{\nu_{\mu}}+\eta_{\nu_{e}}+\eta_{\nu_{\tau}}-\eta_{n} / 2\left(\text { for } \nu_{\mu}\right)
\end{array}
$$

and $\eta^{(\tau)}$ for $\nu_{\tau}$ is obtained from eq. (43) by the interchange $\mu \leftrightarrow \tau$. The individual charge asymmetries, $\eta_{X}$, are defined as the ratio of the difference between particle- 
antiparticle number densities to the number density of photons:

$$
\eta_{X}=\left(N_{X}-N_{\bar{X}}\right) / N_{\gamma}
$$

The first term came from thermal averaging of the time component of the current with which neutrino interacts $\left\langle J_{t}\right\rangle$. This operator is odd with respect to charge conjugation and has different signs for neutrinos and antineutrinos. The second term appears because of non-locality of neutrino interaction related to exchange of intermediate bosons.

The numerical values of these two terms in the effective potential as well as the energy difference, $\delta E=\delta m^{2} / 2 E$, which determines oscillation frequency in vacuum, are

$$
\begin{aligned}
N^{(a)} & =C_{2}^{(a)} G_{F}^{2} T^{4} E / \alpha=5.59 \cdot 10^{-20} C_{2}^{(a)}\left(\frac{T}{\mathrm{MeV}}\right)^{5}\left(\frac{E}{3 T}\right) \mathrm{MeV} \\
A^{(a)} & =C_{1} \eta^{(a)} G_{F} T^{3}=1.166 \cdot 10^{-21} C_{1}\left(\frac{\eta^{(a)}}{10^{-10}}\right)\left(\frac{T}{\mathrm{MeV}}\right)^{3} \mathrm{MeV} \\
\delta E & =\frac{\delta m^{2}}{2 E}=5 \cdot 10^{-13}\left(\frac{\delta m^{2}}{\mathrm{eV}^{2}}\right)\left(\frac{\mathrm{MeV}}{E}\right) \mathrm{MeV}
\end{aligned}
$$

Description of oscillating neutrinos in the early universe in terms of wave function is not adequate because the effects of breaking of coherence by neutrino annihilation or non-forward scattering, as well as neutrino production are essential and one has to use density matrix formalism [2, 45]. Equation for the evolution of the density matrix has the form:

$$
\frac{d \rho}{d t}=\left[\mathcal{H}_{1}, \rho\right]-i\left\{\mathcal{H}_{2}, \rho\right\}
$$

where $\mathcal{H}_{1}$ is the effective Hamiltonian calculated in the first order in $G_{F}$ (see eq. (40) and $\mathcal{H}_{2}$ is the imaginary part of the effective Hamiltonian in the second order in $G_{F}$. Square brackets mean commutator and curly ones mean anticommutator. The last term turns into the usual collision integral in non-oscillating case. In many 
problems this term is approximated as $-\gamma\left(\rho-\rho_{e q}\right)$ where $\gamma$ is the effective strength of interactions and $\rho_{e q}$ is proportional to unit matrix with the coefficient $f_{e q}$, the latter being the equilibrium distribution function. Sometimes this approximation reasonably well describes realistic situation but in many practically interesting cases this is not so and more accurate form of the coherence breaking terms should be taken (see e.g. ref. [1]).

\subsection{Impact of active-sterile oscillations on BBN}

All three mentioned above effects of neutrino influence on BBN may be present in case of mixing of sterile and active neutrinos.

1. Production of $\nu_{s}$ by oscillations may be efficient enough to produce noticeable contribution into total cosmological energy density making $N_{\nu}>3$.

2. If MSW resonance transition [46] is possible a large lepton asymmetry may be developed in the sector of active neutrinos and if it happens to be $\nu_{e}$-asymmetry it may result in a drastic change in primordial abundances of light elements.

3. Oscillations may distort energy spectrum of neutrinos because probability of transformation depends upon their energy.

We will discuss here mostly the first phenomenon because of continuing controversy in the literature (see refs. [1, 6]). Discussion and references to effects of spectral distortion and asymmetry generation can be found in review [1].

Let us assume that there is mixing only between two neutrinos, one active and one sterile, and the MSW resonance condition is not realized (this is so if $\nu_{s}$ is heavier than its active partner, $\delta m^{2}>0$ ). The density matrix is $2 \times 2$ and has 4 elements which satisfy the following kinetic equations:

$$
\dot{\rho}_{s s}=s_{2} \delta E I
$$




$$
\begin{aligned}
\dot{\rho}_{a a} & \left.=-s_{2} \delta E I-\int d \tau\left|A_{e l}\right|^{2}\left[\rho_{a a}\left(p_{1}\right) f\left(p_{2}\right)-\rho_{(} p_{3}\right) f\left(p_{4}\right)\right] \\
& \left.-\int d \tau\left|A_{a n n}\right|^{2}\left[\rho_{a a}\left(p_{1}\right) \bar{\rho}_{(} p_{2}\right)-f\left(p_{3}\right) f\left(p_{4}\right)\right] \\
\dot{R} & =W I-(1 / 2) \Gamma R \\
\dot{I} & =-W R-(1 / 2) \Gamma I+(1 / 2) s_{2} \delta E\left(\rho_{a a}-\rho_{s s}\right)
\end{aligned}
$$

where $R$ and $I$ are real and imaginary parts of the non-diagonal components of the density matrix, $\rho_{a s}=R+i I, \delta E=\delta m^{2} / 2 E, s_{2}=\sin 2 \theta, c_{2}=\cos 2 \theta, W=c_{2} \delta E+V_{e f f}$ (no-resonance condition means that $W \neq 0$ ), and $\Gamma$ is the total interaction rate of neutrinos with all other particles. In Boltzmann approximation it is given by the expression (12) with $D=80\left(1+g_{L}^{2}+g_{R}^{2}\right)$. Fermi corrections are calculated in ref. 47. They diminish the results by $10-15 \%$. Integration in eq. (50) is taken over the phase space of particles 2,3 , and 4 that participate in the reaction $1+2 \leftrightarrow 3+4$.

First we introduce new dimensionless variables, $x$ and $y$, as defined after eq. (11). After that the last two equations (51,52) can be solved analytically as:

$$
\rho_{a s}=\frac{i}{2} \int_{0}^{x} \frac{d x_{1}}{H_{1} x_{1}} s_{2} \delta E\left(\rho_{a a}-\rho_{s s}\right)_{1} \exp \left[-\int_{x_{1}}^{x} \frac{d x_{2}}{H_{2} x_{2}}(i W+\Gamma / 2)_{2}\right]
$$

Here sub-indices 1 and 2 mean that the corresponding functions are taken at $x_{1}$ or at $x_{2}$.

One can estimate that the maximum production rate of sterile neutrinos takes place at the temperature 48

$$
T_{\text {prod }}^{\nu_{s}}=(10-15)(3 / y)^{1 / 3}\left(\delta m^{2} / \mathrm{eV}^{2}\right)^{1 / 6} \mathrm{MeV}
$$

The first number above is for mixing of $\nu_{s}$ with $\nu_{e}$, while the second one is for mixing with $\nu_{\mu}$ or $\nu_{\tau}$. Thus if the neutrino mass difference is not too small the production of sterile neutrinos is efficient when $\Gamma \gg H$. Hence the integrals in eq. (53) are exponentially dominated by upper limits and can be easily taken:

$$
\rho_{a s}=R+i I=\frac{s_{2} \delta E}{2 W-i \Gamma}\left(\rho_{a a}-\rho_{s s}\right)
$$


This is essentially the stationary point approximation which is valid if $\Gamma$ is sufficiently large.

Substituting this result into eq. (49) we find

$$
H x \partial_{x} \rho_{s s}=\frac{\Gamma}{4}\left(\rho_{a a}-\rho_{s s}\right) \frac{s_{2}^{2}}{\left(c_{2}^{2}+V_{e f f} / \delta E\right)^{2}+\Gamma^{2} / 4 \delta E^{2}}
$$

If we neglect $\rho_{s s}$ in comparison with $\rho_{a a}$ and $\Gamma^{2}$ in comparison with $W^{2}$ then we obtain that the rate of production of sterile neutrinos is

$$
\Gamma_{s}=\frac{1}{4} \Gamma_{a} \sin ^{2} 2 \theta_{m}
$$

where $\theta_{m}$ is the effective mixing angle in matter. Its definition is evident from comparison of eqs. (56) and (57). This result is twice smaller than the estimates used in earlier papers 48, 49, 50] and repeated in the recent review [6].

Correspondingly the limit on oscillations parameters becomes weaker:

$$
\begin{aligned}
& \left(\delta m_{\nu_{e} \nu_{s}}^{2} / \mathrm{eV}^{2}\right) \sin ^{4} 2 \theta_{\text {vac }}^{\nu_{e} \nu_{s}}=3.16 \cdot 10^{-5}\left(g_{*}\left(T_{\text {prod }}^{\nu_{s}}\right) / 10.75\right)^{3}\left(\Delta N_{\nu}\right)^{2} \\
& \left(\delta m_{\nu_{\mu} \nu_{s}}^{2} / \mathrm{eV}^{2}\right) \sin ^{4} 2 \theta_{\text {vac }}^{\nu_{\mu} \nu_{s}}=1.74 \cdot 10^{-5}\left(g_{*}\left(T_{\text {prod }}^{\nu_{s}}\right) / 10.75\right)^{3}\left(\Delta N_{\nu}\right)^{2}
\end{aligned}
$$

If $\Delta N$ is not very small, then a better approximation in the bounds above would be $\ln ^{2}(1-\Delta N)$ instead of $(\Delta N)^{2}$.

Possibly these bounds would be even weaker if we take into account that oscillations conserve total number density of neutrinos and the latter can be changed only by the neutrino annihilation into $e^{+} e^{-}$-pairs which froze at rather high temperature (see discussion after eq. (12).

In the resonance case a striking effect of a huge rise of lepton asymmetry of active neutrinos may take place [51] leading to asymmetry close to 1. Due to small fluctuations of baryon asymmetry this huge lepton asymmetry may strongly fluctuate at cosmologically large scales [52]. After neutrino decoupling these inhomogeneities would give rise to significant fluxes of neutrinos. The latter in turn could create 
local electric currents with non-zero vorticity which may be sources of seed magnetic fields [53]. Turbulent eddies generated by such flows could also generate gravitational waves potentially observable in forthcoming LISA mission [54].

\subsection{Oscillations between active neutrinos}

Normally active neutrinos are in thermal equilibrium even at low temperatures and oscillations between them do not lead to any change in their distribution. If however cosmological lepton asymmetry is non-zero and different for different neutrino flavors then the oscillations may change it and lead to equality of all asymmetries. Naively one would expect that in the case of large asymmetry the mixing angle in matter is very strongly suppressed and neutrino flavor transformations are absent. This is not the case however because of a large non-diagonal matrix elements of the effective potential. This was first noticed in ref. [55] and discussed in detail in series of papers [56]. A clear description these phenomena was presented recently in the paper [57.

The kinetic equations used in the previous section can be easily modified to apply to this case. Let us consider for definiteness oscillations between $\nu_{e}$ and $\nu_{\mu}$. One has to take into account the self-interaction processes $\nu_{e} \nu_{\mu} \leftrightarrow \nu_{e} \nu_{\mu}$ and $\nu_{e} \bar{\nu}_{e} \leftrightarrow \nu_{\mu} \bar{\nu}_{\mu}$. The refraction index is determined by the forward scattering amplitude and since $\nu_{e}$ and $\nu_{\mu}$ are considered to be different states of the same particle one has to include both processes when there is a $\nu_{e}$ with momentum $p_{1}$ in initial state and a $\nu_{e}$ or $\nu_{\mu}$ with the same momentum in the final state. The processes of forward transformation $\nu_{e} \leftrightarrow \nu_{\mu}$ give non-diagonal contributions to refraction index. Such transformations always exist, even among non-oscillating particles, but only in the case of non-vanishing mixing the non-diagonal terms in the effective potential become observable. 
Now effective Hamiltonian has the form:

$$
H_{\text {int }}^{(e, \mu)}=\delta E\left(\begin{array}{ll}
h_{e e} & h_{e \mu} \\
h_{\mu e} & h_{\mu \mu}
\end{array}\right) \equiv \frac{\delta E}{2}\left(h_{0}+\sigma \mathbf{h}\right)
$$

where $\delta E=\delta m^{2} / 2 E$ and $\sigma$ are Pauli matrices. The elements of the Hamiltonian matrix (60) are expressed through the integrals over momenta of the distribution functions of other leptons in the plasma and, in particular, of the elements of the density matrix of oscillating neutrinos themselves. The structure of these terms is essentially the same as those discussed above for mixing between active and scalar neutrinos, see eq. (41). The contribution of self-interaction of neutrinos and antineutrinos also contains two terms. One originates from non-locality of weak interactions and is symmetric with respect to charge conjugation:

$$
\mathbf{h}_{+}=\frac{V_{\text {sym }}}{2 \pi^{2}} \int d y y^{3}(\mathbf{P}+\overline{\mathbf{P}}) \text {. }
$$

The second is proportional to the charge asymmetry in the plasma and equals

$$
\mathbf{h}_{-}=\frac{V_{a s y m}}{2 \pi^{2}} \int d y y^{2}(\mathbf{P}-\overline{\mathbf{P}})
$$

An essential feature, specific for oscillations between active neutrinos, is the presence of non-diagonal terms in the Hamiltonian (or in refraction index). In the case of large lepton asymmetry in the sector of oscillating neutrinos, the asymmetric terms in the Hamiltonian strongly dominate and, as a result, the suppression of mixing angle in the medium, found for $\left(\nu_{a}-\nu_{s}\right)$-oscillations, disappears. To be more precise initially the non-diagonal matrix elements are zero but they quickly rise, if the initial asymmetry is not too high, and soon become very large.

A large contribution of lepton asymmetry into effective potential permits to solve kinetic equations for density matrix analytically and find [1]:

$$
h_{z}^{\prime}=-\frac{1}{2} h_{z} \int d^{3} y e^{-y} \frac{s_{2}^{2} \gamma}{\langle\gamma\rangle^{2}+c_{2}^{2}}
$$


where $\gamma=\Gamma / \delta E$ and brackets in the denominator mean averaging over neutrino spectrum.

The solution of this equation is straightforward. It shows that oscillations are not suppressed by matter effects in the presence of large lepton asymmetry. A detailed numerical investigation of oscillations between three active neutrinos in the early universe in presence of a large lepton asymmetry was carried out in the paper [58]. Similar investigation both analytical and numerical was also performed in the papers [59].

An analysis of the impact of oscillating neutrinos on BBN was performed for the values of oscillation parameters favored by the solar and atmospheric neutrino anomalies [58]. For the large mixing angle (LMA) solution flavor equilibrium is established in the early universe and all chemical potentials $\xi_{e, \mu, \tau}$ acquire equal values. The results of the calculations for this case are presented in fig. 6. Since for these values of the parameters, asymmetries in muonic and tauonic sectors are efficiently transformed into electronic asymmetry, the BBN bounds on chemical potentials are quite strong,

$$
\left|\xi_{a}\right|<0.07
$$

for any flavor $a=e, \mu, \tau$.

This result does not destroy the mechanisms of generation of seed magnetic fields and gravitational waves discussed at the end of sec. 6.2. Indeed, the maximum value of chemical potential that could be generated by oscillations is 0.6 and may be factor 2-3 smaller depending on the mixing parameters. If shared between three neutrino species these lower values would not contradict the bound (64). Moreover, for a large region of parameter space the essential rise of asymmetry takes place below neutronproton freezing temperature and does not produce a strong effect on BBN (see e.g. numerical [60] or analytical [61] calculations). 


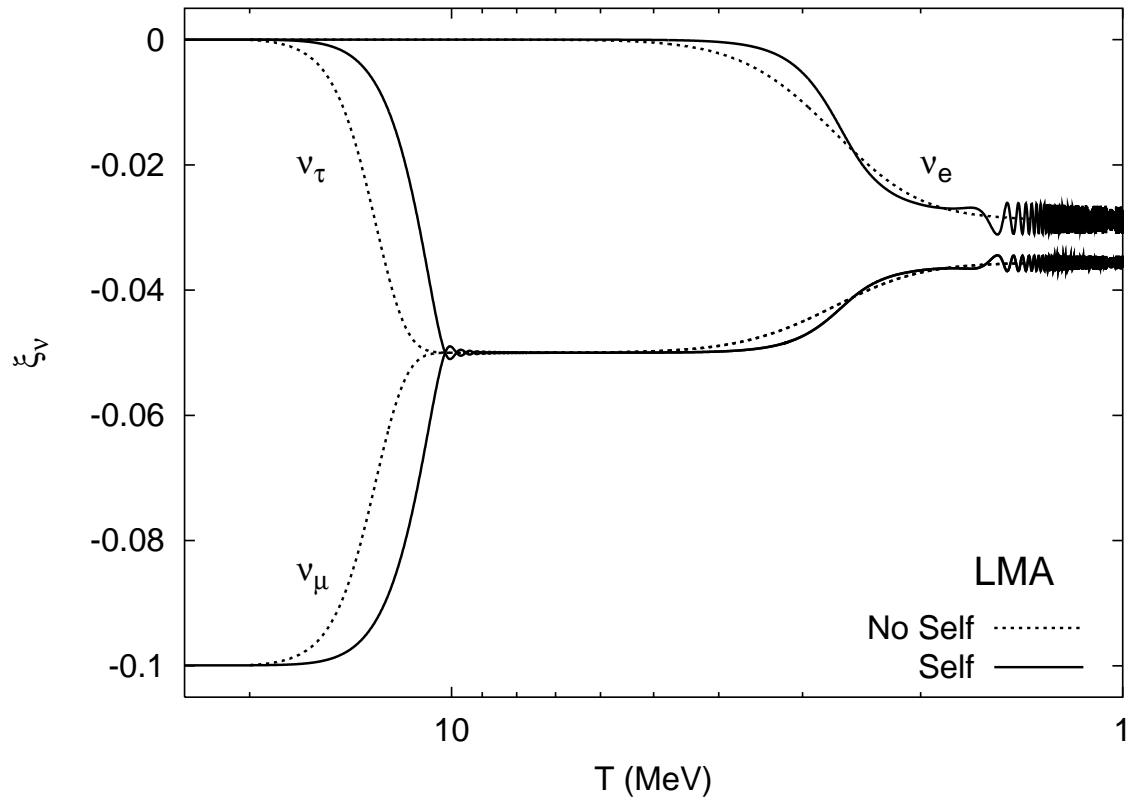

Figure 6: Evolution of neutrino chemical potentials for LMA case, $\theta_{13}=0$, and initial values $\xi_{e}=\xi_{\tau}=0$ and $\xi_{\mu}=-0.1$. Solid and dotted curves are obtained with and without neutrino self-interactions respectively.

For the LOW mixing angle solution the transformation of muon or tauon asymmetries into electronic one is not so efficient. The transformation started at $T<1$ $\mathrm{MeV}$ below interesting range for $\mathrm{BBN}$. The results of calculations are presented in fig. [.

The results presented in these figures are valid for vanishing mixing angle $\theta_{13}$ (in the standard parameterization of the $3 \times 3$-mixing matrix. An analysis of different non-zero values of $\theta_{13}$ can be found in the paper [58].

\section{Acknowledgment}

I am grateful to Yukawa Institute for Theoretical Physics for hospitality during period when the final version of these lectures was prepared. I also thank Daniela Kirilova for helpful comments. 


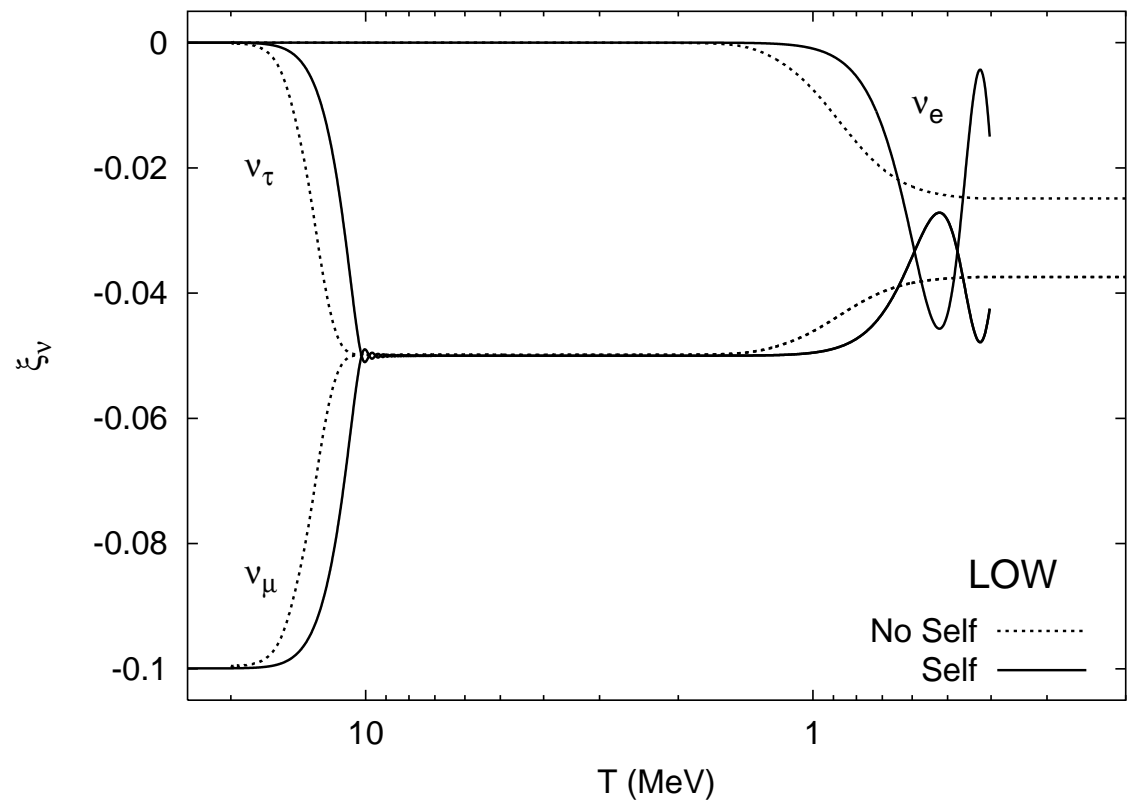

Figure 7: Evolution of the neutrino degeneracy parameters for LOW case and the initial values $\xi_{e}=\xi_{\tau}=0$ and $\xi_{\mu}=-0.1$. Notations are the same as in fig. 6.

\section{References}

[1] A.D. Dolgov, hep-ph/0202122 (to be published in Phys. Repts.).

[2] A.D.Dolgov, Yad.Fiz. 33 (1981) 1309 [Sov. J. Nucl. Phys. 33 (1981) 700].

[3] Particle Data Group, K. Hagiwara et al., Phys. Rev. D66 (2002) 010001.

[4] J.W.F. Valle, hep-ph/0205216;

M. Maltoni, T. Schwetz, M.A. Tortola, J.W.F. Valle, hep-ph/0207157, hepph/0207227.

[5] M.Plionis, astro-ph/0205166;

J. L. Sievers, J. R. Bond, J. K. Cartwright, et al, astro-ph/0205387;

S.E. Deustua, R. Caldwell, P. Garnavich, L. Hui, A. Refregier, astro-ph/0207293. 
[6] K. Kainulainen, K.A. Olive, hep-ph/0206163.

[7] S.S. Gerstein, Ya.B. Zeldovich, Pis'ma ZhETF, 4 (1966) 174 [English translation JETP Letters 4 (1966) 120].

[8] R. Cowsik, J. McClelland, Phys. Rev. Lett. 29 (1972) 669.

[9] S. Tremaine, J.E. Gunn, Phys. Rev. Lett. 42 (1979) 407.

[10] J. Garcia-Bellido, hep-ph/0004188;

F. Bernardeau, S. Colombi, E. Gaztanaga, R. Scoccimarro, astro-ph/0112551, Phys. Repts. (to be published).

[11] E.R. Harrison, Phys. Rev. D1 (1970) 2726;

Ya.B. Zeldovich, Astron. Astrophys. 5 (1970) 84.

[12] R.A.C. Croft, W. Hu, R. Davé, Phys. Rev. Lett. 83 (1999) 1092;

M. Fukugita, G.-C. Liu, N. Sugiyama, Phys. Rev. Lett. 84 (2000) 1082;

A.R. Cooray, Astron. Astrophys. 348 (1999) 31;

E. Gawiser, astro-ph/0005475;

S. Hannestad, astro-ph/0205223.

[13] W. Hu, D.J. Eisenstein, M. Tegmark, Phys. Rev. Lett. 80 (1998) 5255;

D.J. Eisenstein, W. Hu, M. Tegmark, Astrophys. J. 518 (1999) 2.

[14] Ya.B. Zeldovich, Advances of Astronomy and Astrophysics, 3 (1965) 242.

[15] Ya.B. Zeldovich, L.B. Okun, S.B. Pikelner, Uspekhi Fiz. Nauk, 87 (1965) 113.

[16] P. Hut, Phys. Lett. B 69 (1977) 85;

B.W. Lee, S. Weinberg, Phys. Rev. Lett. 39 (1977) 165;

M.I. Vysotsky, A.D. Dolgov, Y.B. Zeldovich, Pisma Zh. Eksp. Teor. Fiz. 26 (1977) 200. 
[17] A.D. Dolgov, Ya.B. Zeldovich, Uspekhi Fiz. Nauk, 130 (1980) 559; Rev. Mod. Phys. 53 (1981) 1;

K.A. Olive, M.S. Turner, Phys. Rev. D 25 (1982) 213.

[18] K. Enqvist, K. Kainulainen, J. Maalampi, Nucl. Phys. B 317 (1989) 647;

K. Enqvist and K. Kainulainen, Phys. Lett. B 264 (1991) 367.

[19] K. Griest, M. Kamionkowski, Phys. Rev. Lett. 64 (1990) 615.

[20] W. Hu, D. Scott, N. Sugiyama, M. J. White, Phys. Rev. D52 (1995) 5498.

[21] E. Gawiser, J. Silk, Phys. Rept. 333 (2000) 24.

[22] J. L. Sievers et al, astro-ph/0205387.

[23] S. Hannestad, Phys. Rev. D64 (2001) 083002.

[24] S. Dodelson, E. Gates, A. Stebbins, Astrophys. J. 467 (1996) 10.

[25] A.T. Lee et al., Astrophys. J. 561 (2001) L1;

N.W. Halverson et al., Astrophys. J. 568 (2002) 38;

C.B. Netterfield et al., Astrophys. J. 571 (2002) 604.

[26] X. Wang, M. Tegmark, M. Zaldarriaga, Phys.Rev. D65 (2002) 123001.

[27] D.A. Dicus, E.W. Kolb, A.M. Gleeson, E.C. Sudarshan, V.L. Teplitz, M.S. Turner, Phys. Rev. D26 (1982) 2694;

M.A. Herrera, S. Hacyan, Astrophys. J. 336 (1989) 539 ; Phys. Fluids, 28 (1985) 3253

N.C. Raha, B. Mitra, Phys. Rev. D44 (1991) 393.

[28] A.D. Dolgov, M. Fukugita, JETP Lett. 56 (1992) 123 [Pisma Zh. Eksp. Teor. Fiz. 56 (1992) 129]; Phys. Rev. D46 (1992) 5378. 
[29] S. Dodelson, M. S. Turner, Phys. Rev. D46 (1992) 3372.

[30] S. Hannestad, J. Madsen, Phys. Rev. D52 (1995) 1764.

[31] A.D. Dolgov, S.H. Hansen, D.V. Semikoz, Nucl. Phys. B503 (1997) 426; Nucl. Phys. B543 (1999) 269.

[32] N.Y. Gnedin, O.Y. Gnedin, Astrophys. J. 509 (1998) 11.

[33] S. Esposito, G. Miele, S. Pastor, M. Peloso, O. Pisanti, Nucl. Phys. B590 (2000) 539.

[34] A. D. Dolgov, Nucl. Phys. B496 (1997) 437.

[35] R.E. Lopez, S. Dodelson, A. Heckler, M.S. Turner, Phys. Rev. Lett. 82 (1999) 3952.

[36] L. Kawano, FERMILAB-PUB-92-04-A.

[37] F. Hoyle, R.J. Tayler, Nature, 203 (1964) 1108.

[38] P.J.E. Peebles, Phys. Rev. Lett. 16 (1966) 410.

[39] V. F. Shvartsman, Pisma Zh. Eksp. Teor. Fiz. 9 (1969) 315 [JETP Lett. 9 (1969) 184].

[40] G. Steigman, D.N. Schramm, J.R. Gunn, Phys. Lett. B 66 (1977) 202.

[41] A.D. Dolgov, Nucl. Phys. B, Proc. Suppl. 110 (2002) 137.

[42] K. Kohri, M. Kawasaki, K. Sato, Astrophys. J. 490 (1997) 72.

[43] S.H. Hansen, G. Mangano, A. Melchiorri, G. Miele, O. Pisanti, Phys. Rev. D65 (2002) 023511. 
[44] D. Nötzold and G. Raffelt, Nucl. Phys. B307 (1988) 924.

[45] L. Stodolsky, Phys. Rev. D36 (1987) 2273;

M.A. Rudzsky, Astrophys. Space Sci. 165 (1990) 65;

M.J. Thomson, B.H.J. McKellar, Univ. of Melbourn preprint, UMP 89-108, Univ. of Manchester preprint, MC-TP-91-1;

G. Raffelt, G. Sigl, L. Stodolsky, Phys. Rev. Lett. 70 (1993) 2363;

G. Sigl and G. Raffelt, Nucl. Phys. B406 (1993) 423.

[46] S.P. Mikheev, A.Yu. Smirnov, Sov. J. Nucl. Phys. 42 (1985) 913 [Yad. Fiz. 42 (1985) 1441]; Nuovo Cim. C9 (1986) 17;

L. Wolfenstein, Phys. Rev. D17 (1978) 2369.

[47] A.D. Dolgov, S.H. Hansen, S. Pastor, D.V. Semikoz, Astropart. Phys. 14 (2000) 79.

[48] R. Barbieri, A.D. Dolgov, Phys. Lett. B237 (1990) 440.

[49] K. Kainulainen, Phys. Lett. B244 (1990) 191.

[50] R. Barbieri, A.D. Dolgov, Nucl. Phys. B349 (1991) 743;

K. Enqvist, K. Kainulainen, J. Maalampi, Nucl. Phys. 349 (1991) 754.

[51] R. Foot, M.J. Thomson, R.R. Volkas, Phys. Rev. D53 (1996) 5349.

[52] P. Di Bari, Phys. Lett. B482 (2000) 150.

[53] A.D. Dolgov, D.Grasso, Phys. Rev. Lett. 88 (2002) 011301.

[54] A.D. Dolgov, D. Grasso, A. Nicolis, astro-ph/0206461.

[55] J. Pantaleone, Phys. Lett. B287 (1992) 128. 
[56] S. Samuel, Phys. Rev. D48 (1993) 1462;

V.A. Kostelecký, J. Pantaleone, S. Samuel, Phys. Lett. B315 (1993) 46;

V. A. Kostelecký, S. Samuel, Phys. Rev. D49 (1994) 1740;

V. A. Kostelecký, S. Samuel, Phys. Rev. D52 (1995) 3184;

S. Samuel, Phys. Rev. D53 (1996) 5382;

V.A. Kostelecký, S. Samuel, Phys. Lett. B385 (1996) 159.

[57] S. Pastor, G.G. Raffelt, D.V. Semikoz, Phys. Rev. D65 (2002) 05301.

[58] A.D. Dolgov, S.H. Hansen, S. Pastor, S.T. Petcov, G.G. Raffelt, D.V. Semikoz, Nucl.Phys. B632 (2002) 363.

[59] C.Lunardini, A.Yu.Smirnov, Phys.Rev. D64 (2001) 073006;

Y.Y.Y. Wong, Phys.Rev. D66 (2002) 025015;

K.N. Abazajian, J.F. Beacom, N.F. Bell, Phys.Rev. D66 (2002) 013008.

[60] P. Di Bari and R. Foot, Phys. Rev. D63 (2001) 043008.

[61] A.D. Dolgov, Nucl. Phys. B610 (2001) 411. 